\title{
Complete response to capecitabine in a frail, elderly patient with metastatic colorectal cancer: A case report
}

\author{
MORENA FASANO $^{1}$, ALESSIO FABOZZI ${ }^{1}$, GUIDO GIORDANO ${ }^{2}$, FILIPPO VENTURINI ${ }^{1}$, GAETANO AURILIO ${ }^{3}$, \\ FLAVIA CANTILE ${ }^{4}$, TERESA FABOZZI ${ }^{5}$, VINCENZO RICCI ${ }^{6}$, GIUSEPPE SANTABARBARA $^{7}$, \\ FLORIANA MORGILLO $^{1}$, FORTUNATO CIARDIELLO ${ }^{1}$ and FERDINANDO DE VITA ${ }^{1}$ \\ ${ }^{1}$ Department of Clinical and Experimental Medicine`F. Magrassi-A. Lanzara’, Division of Medical Oncology, \\ Second University of Naples School of Medicine, I-80131 Naples; ${ }^{2}$ Medical Oncology Unit, San Filippo Neri Hospital, \\ I-00135 Rome; ${ }^{3}$ Urogenital and Head and Neck Cancer Division, European Institute of Oncology, I-20141 Milan; \\ ${ }^{4}$ Department of Oncology and Hematology, Division of Medical Oncology, University Hospital 'Policlinico di Modena', \\ I-41124 Modena; ${ }^{5}$ Department of Solvent Oncology, IRCSS San Raffaele, I-20132 Milan; ${ }^{6}$ Department of Oncology, \\ S. Croce and Carle Teaching Hospital, I-12100 Cuneo; ${ }^{7}$ Division of Medical Oncology, 'San Giuseppe Moscati' Hospital, \\ I-83100 Avellino, Italy
}

Received March 17, 2015; Accepted April 8, 2016

DOI: $10.3892 / 01.2016 .5523$

\begin{abstract}
The clinical management of frail, elderly patients affected by colorectal cancer (CRC) remains a subject of debate. The present study reports the case of an elderly man with metastatic CRC (mCRC) who was successfully treated with capecitabine. The patient survived for 29 months, thus highlighting its potential activity in terms of obtaining a complete response and high efficacy. A 77-year-old man presented with adenocarcinoma of the rectum with multiple and synchronous liver metastases, in addition to several comorbidities. The patient received single-agent capecitabine chemotherapy (825 mg/mq twice a day) on days 1-14 of a 21-day cycle. Following 12 cycles of well-tolerated therapy, a computed tomography scan revealed a complete response with no evidence of liver metastases. An overall survival of 29 months was documented, and the patient eventually succumbed to a diabetes-related complication. In compromised patients with $\mathrm{mCRC}$, reduced-dose capecitabine is an excellent therapeutic option due to its positive safety profile, activity and efficacy.
\end{abstract}

\section{Introduction}

Colorectal cancer (CRC) represents the second highest cause of cancer-associated mortality in developed countries and is

Correspondence to: Dr Alessio Fabozzi, Department of Clinical and Experimental Medicine‘F. Magrassi-A. Lanzara', Division of Medical Oncology, Second University of Naples School of Medicine, II Policlinico, 5 Pansini Street, I-80131 Naples, Italy

E-mail: alessiofabozzi@gmail.com

Key words: colorectal cancer, frail elderly patient, capecitabine, complete response, advanced disease responsible for $\sim 530,000$ fatalities each year worldwide $(1,2)$. Furthermore, CRC is the third most commonly diagnosed malignant tumor, with more than 1,000,000 new cases every year globally (3). The 5-year survival rate is estimated at $65 \%$ in North America and $54 \%$ in Western Europe $(1,4)$. The incidence of colorectal cancer is uncommon under the age of 50 years, predominantly in tumors induced by heredity and with a family history, but the risk increases to the age of $\sim 85$ years. The incidence of colorectal adenomas also rises with age. Indeed, two-thirds of all colorectal cancers occurred in patients over the age of 65 (5).

Regarding CRC management, comorbidities should be taken into consideration, particularly in elderly patients, as their incidence increases with age and strongly affects patient prognosis $(6,7)$. For example, a history of ischemic heart disease may limit the employment of drugs, such as bevacizumab, considering its association with the development of ischemic heart disease $(0.52-1.7 \%$ of the treated population) (8). Furthermore, this suggests a particular caution is required for the use of 5-fluorouracil (5-FU) and capecitabine, which can induce cardiotoxicity in $0-35 \%$ of the treated population, which often presents as myocardial ischemia, but to a lesser extent cardiac arrhythmias, hyper and hypotension, left ventricular dysfunction, cardiac arrest and sudden death (9). However, comorbidities are not the only factor to consider when planning CRC management. Functional status is also widely known to affect patient survival and treatment tolerance in oncology. Geriatric studies have reported that patients with functional limitations are at higher risk of functional decline or mortality over 2 years after functional limitations development compared with their more functional counterparts (10). Frail, elderly patients are often easily identifiable, and the treatment approach is primarily palliative. This may occasionally involve the cautious use of chemotherapy (11).

The present study describes the case of an elderly man with metastatic CRC (mCRC) who was successfully treated 


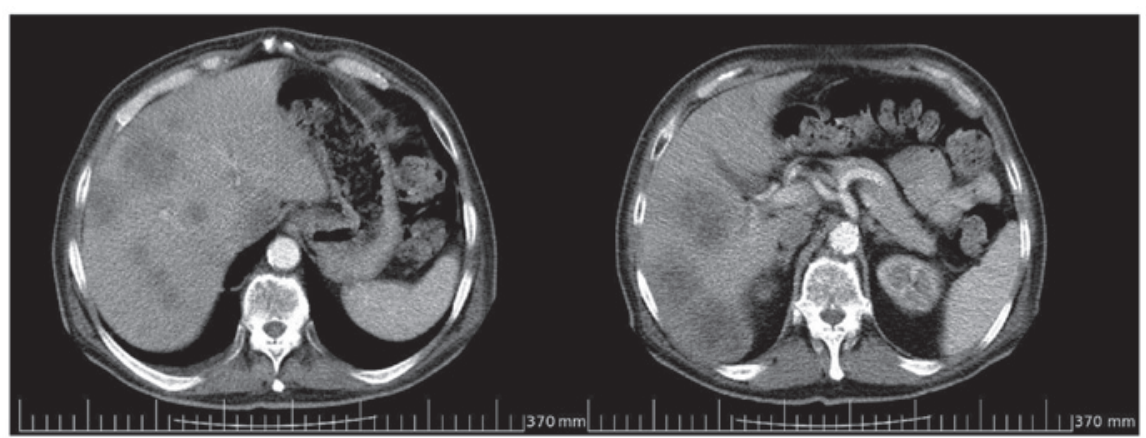

Figure 1. Contrast-enhanced computed tomography scan performed in May 2008 showing evidence of multiple, isodense, solid nodules in the left and right liver lobes.

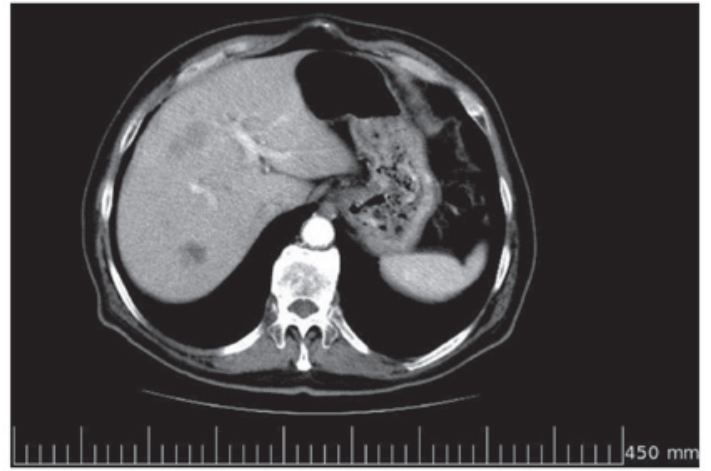

Figure 2. Contrast-enhanced computed tomography scan performed in January 2009 showing a reduction of $>75 \%$ in the number and size of the multiple, solid lesions evidenced in each liver lobe in previous examinations

with single-agent capecitabine chemotherapy, underlining the positive activity of the drug in terms of safety and obtaining a complete response.

\section{Case report}

In September 2008, a 77-year-old man was referred to our department following an anterior rectal-sigmoid resection performed at Surgical Division of Second University of Naples, with a histological confirmation of adenocarcinoma of the rectum and multiple liver metastases. At diagnosis, the Eastern Cooperative Oncology Group (ECOG) (12) performance status score was 2. Excluding the presence of cancer, the medical history of the patient included the following conditions: Type II diabetes complicated by retinopathy with loss of vision, skin ulcers and arterial disease, which was treated with insulin; arterial hypertension, which was treated with angiotensin-converting-enzyme inhibitors and calcium antagonists, but was poorly controlled; transitory ischemic attack (TIA), which occurred in 2006 during acetylsalicylic acid treatment; and hyperlipidemia, which occurred during treatment with statins.

In May 2008, due to severe abdominal pain in the left iliac region, weight loss (8 $\mathrm{kg}$ in 3 months) and anemia (hemoglobin level, $9.2 \mathrm{~g} / \mathrm{dl}$; normal range, 13.5 -17 g/dl in the male population), the patient underwent abdominal ultrasonography, which revealed multiple, hyperechoic nodules with the maximum diameter of $3.5 \mathrm{~cm}$ in the right

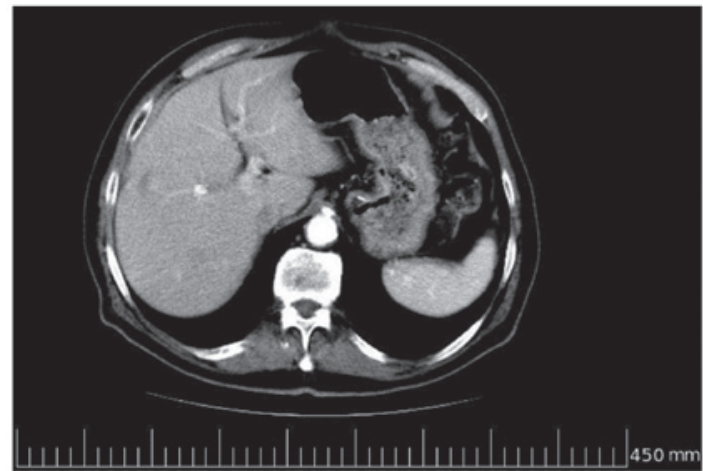

Figure 3. Contrast-enhanced computed tomography scan performed in June 2009 showing a complete response with no evidence of liver metastases.

and left liver lobes. Subsequently, a computed tomography (CT) scan confirmed abnormal liver density, the presence of multiple, solid nodules in the left and right lobes, and wall thickening of the sigmoid colon (Fig. 1). These findings required further evaluation via colonoscopy examination. The colonoscopy was conducted up until the rectosigmoid junction due to an intestinal constriction caused by a large lesion with the maxium diameter of $4 \mathrm{~cm}$, partially occluding the intestinal lumen, ulcerated and bleeding when touched; two biopsies were performed, which were each positive for moderately differentiated (G2) ulcerated adenocarcinoma. Laboratory tests were performed and the tumor marker levels were as follows: Carcinoembryonic antigen (CEA), $337.2 \mathrm{ng} / \mathrm{ml}$ (normal range, 0-5 ng/ml); carbohydrate antigen (CA)19-9, $109.7 \mathrm{U} / \mathrm{ml}$ (normal range 0-45 U/ml); and CA125, 98.2 U/ml (normal range: 0-35 U/ml). In June 2008, the patient underwent resection of the sigmoid tract due to bowel obstruction. Histological examination was indicative of moderately-differentiated (G2) ulcerated adenocarcinoma, chromogranin negative, with stromal desmoplasia and invasion of the intestinal wall to the adipose tissue of the root mesenteric artery. A total of 16 lymph nodes were resected and metastasis from adenocarcinoma was identified in 4 of them. According to the tumor-node-metastasis classification, the adenocarcinoma was staged as pT3N2M1 G2 (13). Due to a post-surgical delay, the patient underwent observation in the Department of Clinical and Experimental Medicine 'F. Magrassi-A. Lanzara', Division of Medical Oncology, Second University of Naples School of Medicine 


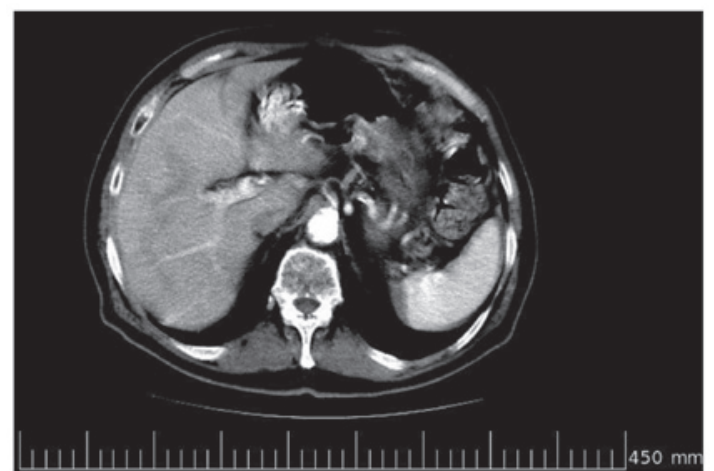

Figure 4. Contrast-enhanced computed tomography scan performed in July 2010 confirming a complete response and no evidence of liver metastases.

in September 2008. Considering the presence of several comorbidities and according to the clinical condition of the patient, single-agent treatment with oral capecitabine was administered at the reduced dose of $825 \mathrm{mg} / \mathrm{mq}$ twice a day, for a total dose of 3,000 mg/day, on days 1-14 every 3 weeks (the standard dose routinely employed in our department was $1,250 \mathrm{mg} / \mathrm{mq}$ twice a day on days $1-14$ every 3 weeks).

In January 2009, at the end of the sixth chemotherapy cycle, a contrast-enhanced CT scan revealed a reduction of $>75 \%$ in the number and size of the multiple, solid lesions evidenced in each liver lobe in the previous examinations (Fig. 2). Further tumor marker laboratory tests confirmed this response, indicating significantly decreased levels compared with the pre-operative baselines (CEA, $7.2 \mathrm{ng} / \mathrm{ml}$ vs. $337.2 \mathrm{ng} / \mathrm{ml}$; CA19-9, $7.3 \mathrm{U} / \mathrm{ml}$ vs. $109.7 \mathrm{U} / \mathrm{ml}$; CA125, $31.3 \mathrm{U} / \mathrm{ml}$ vs. $98.2 \mathrm{U} / \mathrm{ml})$. No hematological, gastrointestinal and/or hand-foot syndrome toxicity was recorded. This response, in addition to the positive tolerability and safety of the treatment resulted in the continuation of the therapy. In June 2009, after 6 further cycles of the chemotherapy, a further total-body contrast-enhanced CT scan was performed, which revealed evidence of a complete response, with no evidence of liver metastases (Fig. 3). Due to the length of treatment and the evidence of a complete response, chemotherapy was discontinued, whilst clinical-instrument examination was continued with contrast-enhanced CT every 6 months, abdominal ultrasound examination and annual colonoscopy and laboratory haematological examination and tumor marker level assessment (CEA, CA19.9 and CA125) every 3 months. In July 2010, a contrast-enhanced CT scan confirmed the complete response (Fig. 4). However, the patient succumbed to a diabetes-related complication in October 2010 following 29 months of overall survival. Ethical approval for the publication on the present case report was obtained from the patient's family.

\section{Discussion}

Approximately $20 \%$ of patients with CRC present with an advanced stage of the disease at diagnosis $(1,2,4)$. For this reason, prolongation of progression-free survival, palliation of symptoms, improvement of quality of life, and only rarely, complete recovery, represent the most feasible targets that may be pursued in advanced-stage patients. Several lines of evidence have indicated that patients with $\mathrm{mCRC}$ benefit, in terms of survival, from the three most active chemotherapeutic agents [leucovorin (LV)/5-FU, irinotecan and oxaliplatin] dispensed either sequentially or concomitantly (14-16). However, the current literature does not offer enough data regarding the use of chemotherapy in frail, elderly patients with mCRC.

A primary concern raised by the present study is how the term 'elderly' is defined. Clinical trial settings consider the appropriate cut-off age of an elderly individual as $\geq 70$ years (17); however, daily practice has demonstrated that individuals $>70$ years may often have less clinical conditions and a better metabolic balance than younger individuals. Taking this into account, it may be more appropriate to discuss patients in terms of being 'fit' or 'unfit'. Balducci and Extermann (18) performed a comprehensive geriatric assessment that examined facets of health and functionality, which resulted in the identification of three relevant categories: i) 'Fit', ii) 'intermediate' and iii) 'frail' patients. It was determined that fit, elderly patients could be treated with the same schedules used in younger patients. Notably, this subgroup could benefit from first-line strategies, such as the folinic acid, 5-FU and oxaliplatin regimen, and the folinic acid, 5-FU and irinotecan regimen, while the feasibility of adding targeted agents, including cetuximab and bevacizumab, requires further investigation.

In the present case, the choice of a chemotherapeutical regimen using capecitabine as a single agent was supported by several parameters, including the age of the patient, the presence of comorbidities and the current life expectancy. Capecitabine is a third-generation, oral prodrug of 5-FU, produced to closely simulate prolonged intravenous administration of 5-FU (19). Previous studies have demonstrated that it is an effective and well-tolerated first-line monotherapy in elderly patients with $\mathrm{CRC}$, and it has become a valid alternative to LV/5-FU-based combinations, also in association with irinotecan or oxaliplatin $(20,21)$.

In the present study, the decision to take a chemotherapeutical approach with a low dose of capecitabine $(850 \mathrm{mg} / \mathrm{mq}$ twice a day) was due to the presence of type II diabetes complicated by retinopathy with loss of vision, skin ulcers and arterial disease. This decision was supported by the study by Cripps et al (22), which demonstrated that unfit, elderly patients may benefit from a lower dose of capecitabine compared with the standard dose, due to a potentially lower incidence of toxicities. In particular, Jung et al showed that a metronomic dose of capecitabine, with a dose ranging between 1,000 and 2,000 mg daily, without interruption in elderly patients for whom combination chemotherapy or even monotherapy is not feasible, offers a good toxicity profile and good tumor control (23). In a large observational study, 1,249 elderly patients received oral capecitabine as single agent or in combination with other chemotherapeutical agents in first line treatment: Capecitabine-based combination was administered in $56 \%$ of patients in the overall population. The median treatment duration was $\sim 5$ months. Severe toxicity occurred rarely, without any difference regarding age groups. The most common hematological toxicity was anemia. Gastrointestinal side effects and hand-food-syndrome were 
the most frequent non-hematological toxicities. The overall response rate (ORR) was significantly increased in the patient group $\leq 75$ years compared to patients $>75$ years of age (38 vs. $32 \%, \mathrm{p}=0.019)$. Median progression free survival (PFS; 9.7 vs. 8.2 months, $\mathrm{p}=0.00021$ ) and overall survival (OS; 31.0 vs. 22.6 months, $\mathrm{p}<0.0001$ ) was decreased in elderly patients (24). In a phase II trial of 51 patients aged $>70$ years with advanced CRC, capecitabine was effective and well tolerated, with an ORR of $24 \%$, PFS of 7 months and OS of 11 months. Grade 3/4 adverse events were observed in $12 \%$ of patients (25).

The current study considered K-Ras mutational status evaluation and the use of cetuximab to be unnecessary, as this would have required biweekly access to the day hospital, which was not recommended in this frail patient. Bevacizumab was also not recommended due to the possibility of arterial hypertension and the medical history of TIA.

Treatment was well tolerated in the present case with no evidence of grade 3-4 toxicity, and the patient exhibited good compliance to oral administration. These conditions contributed to a complete response of the liver metastases and an overall survival time of 29 months. The literature suggests that the median overall survival time for patients with mCRC, following combinational or sequential use of active drugs and molecules, is $\sim 24$ months (26).

The present study evaluated the incidence and severity of adverse events in elderly patients compared with younger patients through an analysis of the literature. Previous studies have reported that elderly patients have a similar incidence rate of drug-related toxicities compared with younger patients (20-22), probably due to lower functional reserve of single organ. A previous study conducted by ECOG analyzed drug toxicities in 19 trials of advanced cancer in different sites, including CRC. Of the 1,210 cases analyzed, including 174 patients who were $\geq 70$ years, toxicities were uncommon and no differences were observed across the cut-off age. However, the rate of toxicity increased in frail patients (27). Similarly, Cascinu et al (28) did not identify any differences in toxicity in a study of 120 patients with advanced cancer at 6 different sites, including CRC, using a cut-off age of 70 years.

In conclusion, the present case confirms the efficacy of capecitabine chemotherapy in elderly patients with $\mathrm{mCRC}$, even when used as a single agent. The current case indicated that advanced age alone is not a sufficient reason to withhold or limit treatment, including chemotherapy. The possibility that lifestyle and the presence of comorbidities may increase the biological age of a patient should also be taken into account when planning treatment. Chemotherapy is hazardous in patients with a poor performance status, therefore therapeutic choices must be modified case by case. However, the use of chemotherapy in the fit, elderly population may be feasible without increasing either mortality or morbidity, whilst also achieving similar side effects and response rates as observed in younger patients.

\section{References}

1. Jemal A, Murray T, Ward E, Samuels A, Tiwari RC, Ghafoor A, Feuer EJ and Thun MJ: Cancer statistics, 2005. CA Cancer J Clin 55: 10-30, 2005.
2. Mitry E, Bouvier AM, Esteve J and Faivre J: Improvement in colorectal cancer survival: A population-based study. Eur J Cancer 41: 2297-2303, 2005.

3. Rougier P and Mitry E: Epidemiology, treatment and chemoprevention in colorectal cancer. Ann Oncol 14 (Suppl 2): ii3-ii5, 2003.

4. Grothey A, Sargent D, Goldberg RM and Schmoll HJ: Survival of patients with advanced colorectal cancer improves with the availability of fluorouracil-leucovorin, irinotecan, and oxaliplatin in the course of treatment. J Clin Oncol 22: 1209-1214, 2004.

5. Holt PR, Kozuch P and Mewar S: Colon cancer and the elderly: From screening to treatment in management of GI disease in the elderly. Best Pract Res Clin Gastroenterol 23: 889-907, 2009.

6. Extermann M, Overcash J, Lyman GH, Parr J and Balducci L: Comorbidity and functional status are independent in older cancer patients. J Clin Oncol 16: 1582-1587, 1998.

7. Meyerhardt JA, Catalano PJ, Haller DG, Mayer RJ, Macdonald JS, Benson AB III and Fuchs CS: Impact of diabetes mellitus on outcomes in patients with colon cancer. J Clin Oncol 21: 433-440, 2003.

8. Chen XL, Lei YH, Liu CF, Yang QF, Zuo PY, Liu CY, Chen CZ and Liu YW: Angiogenesis inhibitor bevacizumab increases the risk of ischemic heart disease associated with chemotherapy: A meta-analysis. PLoS One 8: e66721, 2013

9. Polk A, Vistisen K, Vaage-Nilsen M and Nielsen DL: A systematic review of the pathophysiology of 5-fluorouracil-induced cardiotoxicity. BMC Pharmacol Toxicol 15: 47, 2014.

10. Saliba D, Elliott M, Rubenstein LZ, Solomon DH, Young RT, Kamberg CJ, Roth C, MacLean CH, Shekelle PG, Sloss EM and Wenger NS: The Vulnerable Elders Survey: A tool for identifying vulnerable older people in the community. J Am Geriatr Soc 49: 1691-1699, 2001.

11. Fried LP, Tangen CM, Walston J, Newman AB, Hirsch C, Gottdiener J, Seeman T, Tracy R, Kop WJ, Burke G, et al; Cardiovascular Health Study Collaborative Research Group: Frailty in older adults: Evidence for a phenotype. J Gerontol A Biol Sci Med Sci 56: M146-M156, 2001

12. Oken MM, Creech RH, Tormey DC, Horton J, Davis TE, McFadden ET and Carbone PP: Toxicity and response criteria of the Eastern Cooperative Oncology Group. Am J Clin Oncol 5: 649-655, 1982.

13. Lan YT, Yang SH, Chang SC, Liang WY, Li AF, Wang HS, Jiang JK, Chen WS, Lin TC and Lin JK: Analysis of the seventh edition of American Joint Committee on colon cancer staging. Int J Colorectal Dis 27: 657-663, 2012.

14. Grothey A and Goldberg RM: A review of oxaliplatin and its clinical use in colorectal cancer. Expert Opin Pharmacother 5: 2159-2170, 2004.

15. Mohelnikova-Duchonova B, Melichar B and Soucek P: FOLFOX/FOLFIRI pharmacogenetics: The call for a personalized approach in colorectal cancer therapy. World J Gastroenterol 20: 10316-10330, 2014.

16. Edwards MS, Chadda SD, Zhao Z, Barber BL and Sykes DP: A systematic review of treatment guidelines for metastatic colorectal cancer. Colorectal Dis 14: e31-e47, 2012.

17. Extermann M, Albrand G, Chen H, Zanetta S, Schonwetter R, Zulian GB, Cantor A and Droz JP: Are older French patients as willing as older American patients to undertake chemotherapy? J Clin Oncol 21: 3214-3219, 2003.

18. Balducci L and Extermann M: Management of cancer in the older person: A practical approach. Oncologist 5: 224-237, 2000.

19. Wang F, Wang FH, Bai L and Xu RH: Role of capecitabine in treating metastatic colorectal cancer in Chinese patients. Onco Targets Ther 7: 501-511, 2014.

20. Chu E, Eng C, Abbruzzese J, et al: Efficacy and safety of capecitabine for colorectal cancer. Am J Oncol Rev 2 (Suppl 3): $1-28,2003$.

21. Escudero P, Verge V, Feliu J, Sanz-Lacalle J, Lopez R, Gomez MJ, Aparicio A, Yubero A, Lorenzo A and Gonzalez-Baron M: A study of capecitabine in elderly patients as first line treatment in advanced or metastatic colorectal cancer. Proc Am Soc Clin Oncol 22: abstract 3114, 2003.

22. Cripps MC, Vincent M, Jonker D, Kerr I, Dingle B, Martin LA, Mathews J, Biagi J, Knight G and Lam W: Dose reduced first line capecitabine monotherapy in older and less fit patients with advanced colorectal cancer (ACRC). J Clin Oncol 23: 3577, 2005 . 
23. Jung YH, Lee WJ, Byeon JH, Lee IK, Han CW and Woo IS Metronomic chemotherapy with capecitabine for metastatic colorectal cancer in very elderly patients. Korean J Intern Med: Mar 10, 2016 (Epub ahead of print)

24. Stein A, Quidde J, Schröder JK, Göhler T, Tschechne B, Valdix AR, Höffkes HG, Schirrmacher-Memmel S, Wohlfarth T, Hinke A, Engelen A and Arnold D: Capecitabine in the routine first-line treatment of elderly patients with advanced colorectal cancer - results from a non-interventional observation study. BMC Cancer 16: 82, 2016.

25. Feliu J, Escudero P, Llosa F, Bolaños M, Vicent JM, Yubero A, Sanz-Lacalle JJ, Lopez R, Lopez-Gómez L, Casado E, Gómez-Reina MJ and González-Baron M: Capecitabine as firstline treatment for patients older than 70 years with metastatic colorectal cancer: An oncopaz cooperative group study. J Clin Oncol 23: 3104-3111, 2005
26. Ohhara Y, Fukuda N, Takeuchi S, Honma R, Shimizu Y, Kinoshita I, Dosaka-Akita H: Role of targeted therapy in metastatic colorectal cancer. World J Gastrointest Oncol 8: 642-655, 2016.

27. Begg CB and Carbone PP: Clinical trials and drug toxicity in the elderly: The experience of the Eastern Cooperative Oncology Group. Cancer 52: 1986-1992, 1983.

28. Cascinu S, Del Ferro E and Catalano G: Toxicity and therapeutic response to chemotherapy in patients aged 70 years or older with advanced cancer. Am J Clin Oncol 19: 371-374, 1996. 